\title{
UNIFORM REPRESENTATIONS OF THE INCOMPLETE BETA FUNCTION IN TERMS OF ELEMENTARY FUNCTIONS*
}

\author{
CHELO FERREIRA ${ }^{\dagger}$, JOSÉ L. LÓPEZ ${ }^{\ddagger}$, AND ESTER PÉREZ SINUSíA ${ }^{\dagger}$
}

\begin{abstract}
We consider the incomplete beta function $B_{z}(a, b)$ in the maximum domain of analyticity of its three variables: $a, b, z \in \mathbb{C},-a \notin \mathbb{N}, z \notin[1, \infty)$. For $\Re b \leq 1$ we derive a convergent expansion of $z^{-a} B_{z}(a, b)$ in terms of the function $(1-z)^{b}$ and of rational functions of $z$ that is uniformly valid for $z$ in any compact set in $\mathbb{C} \backslash[1, \infty)$. When $-b \in \mathbb{N} \cup\{0\}$, the expansion also contains a logarithmic term of the form $\log (1-z)$. For $\Re b \geq 1$ we derive a convergent expansion of $z^{-a}(1-z)^{b} B_{z}(a, b)$ in terms of the function $(1-z)^{b}$ and of rational functions of $z$ that is uniformly valid for $z$ in any compact set in the exterior of the circle $|z-1|=r$ for arbitrary $r>0$. The expansions are accompanied by realistic error bounds. Some numerical experiments show the accuracy of the approximations.
\end{abstract}

Key words. incomplete beta function, convergent expansions, uniform expansions

AMS subject classifications. 33B20, 41A58, 41A80

1. Introduction. We may find in the literature a large variety of convergent or asymptotic expansions of the special functions of mathematical physics that have the important property of being given in terms of elementary functions: direct or inverse powers of a certain complex variable $z$ and, sometimes, other elementary functions of $z$. However, quite often, these expansions are not simultaneously valid for small and large values of $|z|$. Thus, it would be interesting to derive new convergent expansions of these functions in terms of elementary functions that hold uniformly in $z$ in a large region of the complex plane that includes small and large values of $|z|$.

In $[1,5]$, the authors derived new uniform convergent expansions of the incomplete gamma function $\gamma(a, z)$ and the Bessel functions $J_{\nu}(z)$ and $Y_{\nu}(z)$ in terms of elementary functions of $z$ that hold uniformly in unbounded regions of $\mathbb{C}$ containing the point $z=0$. The starting point of the technique used in $[1,5]$ is an appropriate integral representation of these functions. The key point is the use of a Taylor expansion, at an appropriate point of the integration interval, of a certain factor of the integrand that is independent of the variable $z$. This fact, the independence of this factor with respect to $z$, translates into a convergent uniform expansion in a large region of the complex $z$-plane. The expansions given in $[1,5]$ are accompanied by error bounds and numerical experiments showing the accuracy of the approximations.

In this work, we continue that line of investigation considering the incomplete beta function $B_{z}(a, b)$. This function is used extensively in statistics as the probability integral of the beta distribution and as a special case of the (negative) binomial distribution, Student's distribution, and the $F$-(variance-ratio)distribution [3]. Among its physical applications, we mention its use in Monte Carlo simulations in statistical mechanics [4] and in cosmology [2]. We consider $B_{z}(a, b)$ as a function of the complex variable $z$ and derive new convergent expansions that are uniformly valid in an unbounded region of the complex $z$-plane that contains the point $z=0$. The starting point is the integral definition of the incomplete beta

\footnotetext{
*Received March 22, 2018. Accepted October 16, 2018. Published online on December 4, 2018. Recommended by F. Marcellan.

† Departamento de Matemática Aplicada, IUMA, Universidad de Zaragoza, 50009 Zaragoza, Spain ( $\{$ cferrei, ester.perez\} @unizar.es).

${ }^{\ddagger}$ Departamento de Estadística, Informática y Matemáticas, Universidad Pública de Navarra, 31006 Pamplona, Spain (jl.lopez@unavarra.es).
} 
function [9, eq. 8.17.1],

$$
z^{-a} B_{z}(a, b):=\int_{0}^{1} t^{a-1}(1-z t)^{b-1} d t,
$$

valid for $\Re a>0$ and $z \in \mathbb{C} \backslash[1, \infty)$. The incomplete beta function $B_{z}(a, b)$ reduces to the ordinary beta function $B(a, b)$ when $z=1$ and, except for positive integer values of $b$, has a branch cut discontinuity in the complex $z$-plane running from 1 to $\infty$. When $a$ or $b$ are positive integers, the incomplete beta function is an elementary function of $z$.

For reasons that will become clear later, it is convenient to consider the integral (1.1) only for $\Re b \leq 1$. When $\Re b \geq 1$, we consider instead the following integral representation of $B_{z}(a, b)$ that may be obtained from (1.1) after the change of variable $t \rightarrow 1-t$ :

$$
z^{-a} B_{z}(a, b)=(1-z)^{b-1} \int_{0}^{1}(1-t)^{a-1}\left(1+\frac{z}{1-z} t\right)^{b-1} d t
$$

valid for $\Re a>0$ and $z \in \mathbb{C} \backslash[1, \infty)$.

By using the recurrence relation [9, eq. 8.17.20],

$$
B_{z}(a, b)=\frac{a+b}{a} B_{z}(a+1, b)+\frac{z^{a}(1-z)^{b}}{a},
$$

we find that the function $B_{z}(a, b)$ may be analytically continued in the complex variable $a$ to the negative half plane $\Re a \leq 0$ with poles at the negative integers $a=-1,-2,-3, \ldots$ And conversely, by using repeatedly this formula, we have that $B_{z}(a, b)$, with $\Re a \leq 0$, may be written as a linear combination of elementary functions of its three variables and an incomplete beta function with $\Re a>0$. Therefore, without loss of generality, in the remaining of the paper we restrict ourselves to $\Re a>0$.

The power series expansion of the incomplete Beta function is given by [10]

$$
z^{-a} B_{z}(a, b)=\sum_{n=0}^{\infty} \frac{(1-b)_{n}}{n !(a+n)} z^{n}, \quad|z|<1 .
$$

This expansion also converges absolutely when $|z|=1$ if $\Re b>0$. It may be derived from the integral representation (1.1) by replacing the factor $(1-z t)^{b-1}$ by its Taylor series at the origin and interchanging the order of summation and integration. This Taylor series expansion converges for $t \in[0,1]$, but the convergence is not uniform in $|z|$. Therefore, the expansion (1.3) is not uniform in $|z|$ as the remainder is unbounded when $|z| \rightarrow \infty$.

From the hypergeometric function representation of $B_{z}(a, b)$ [9, eq. (8.17.7)],

$$
B_{z}(a, b)=\frac{z^{a}}{a}{ }_{2} F_{1}(a, 1-b ; a+1 ; z),
$$

and by combining the formulas [8, eq. (15.2.2)] and [8, eqs. (15.8.2) and (15.8.8)], we obtain, for $1-a-b \notin \mathbb{N} \cup\{0\}$ and $|\operatorname{ph}(-z)|<\pi(\operatorname{ph}(z)$ denotes the phase of the complex number $z)$, the asymptotic expansion

$$
\begin{aligned}
z^{-a} B_{z}(a, b) \sim & \frac{\pi \Gamma(a)}{\Gamma(a+b) \sin [\pi(1-a-b)]} \\
& \times\left[\frac{(-z)^{-a}}{\Gamma(1-b)}-\frac{(-z)^{b-1}}{\Gamma(a) \Gamma(1-a-b)} \sum_{k=0}^{\infty} \frac{(1-b)_{k}}{(1-a-b-k) k ! z^{k}}\right] .
\end{aligned}
$$




\section{ETNA}

Kent State University and

Johann Radon Institute (RICAM)

On the other hand, if $1-a-b \in \mathbb{N} \cup\{0\},|z|>1$, and $|\operatorname{ph}(-z)|<\pi$, then we have

$$
\begin{aligned}
& z^{-a} B_{z}(a, b) \sim \frac{\Gamma(a)(-z)^{-a}}{\Gamma(1-b)} \sum_{k=0}^{-a-b} \frac{(a)_{k}(-a-b-k) !}{k ! \Gamma(1-k) z^{k}} \\
&+(-z)^{-a} \sum_{k=0}^{\infty} \frac{(-1)^{k}(1-b)_{k}}{k !(k+1-a-b) ! \Gamma(a+b-k) z^{k+1-a-b}} \\
& \times[\log (-z)+\psi(k+1)+\psi(k+2-a-b) \\
&-\psi(1-b+k)-\psi(a+b-k)],
\end{aligned}
$$

where $\psi$ denotes the digamma function. The expansions (1.4) and (1.5) are asymptotic expansions of the incomplete beta function for large $|z|$, but the remainders are unbounded when $|z| \rightarrow 0$, and hence, these expansions are not uniform in $|z|$ either. Other large parameter asymptotic approximations with certain uniformity properties with respect to the parameters can be found in $[7,11]$.

The expansions (1.3), (1.4), and (1.5) have the good property of being given in terms of elementary functions of $z$, but they have the inconvenience of not being uniform in $|z|$ in unbounded regions of the complex plane that include the point $z=0$. In this paper we show that it is possible to derive convergent expansions of $B_{z}(a, b)$ in terms of elementary functions that hold uniformly for $z$ in an unbounded region of $\mathbb{C}$ that includes the point $z=0$. As an illustration of the approximations that we are going to obtain (see Theorem 2.1 below), we derive, for example, the following one:

$$
\frac{1}{z^{5 / 2}} B_{z}\left(\frac{5}{2}, \frac{1}{2}\right)=\frac{\left(32+40 z-5 z^{2}\right)-\left(27 z^{2}+56 z+32\right) \sqrt{1-z}}{40 \sqrt{2} z^{3}}+\epsilon(z),
$$

with $|\epsilon(z)|<0.0089$ in the negative half plane $\Re z \leq 0$. When $z=0$, the right-hand side of (1.6) must be understood in the limit sense.

In order to derive these kinds of approximations, we use in this paper the technique proposed in [1,5]: we consider a Taylor expansion of the factor $t^{a-1}$ in (1.1) and of the factor $(1-t)^{a-1}$ in (1.2). The factor $t^{a-1}$ in (1.1) is not analytic at the origin unless $a \in \mathbb{N}$ (analogously, the factor $(1-t)^{a-1}$ in (1.2) is not analytic at $\left.t=1\right)$. Following the arguments given in [5], we must consider the expansion of the factors $t^{a-1}$ and $(1-t)^{a-1}$ at the middle point $t=1 / 2$ of the integration interval $(0,1)$ in the respective integrals (1.1) and (1.2) in such a way that we assure that the integration interval is contained in the disk of convergence of the Taylor series. This Taylor expansion is convergent for any $t$ in the integration interval of (1.1) or (1.2), and obviously, it is independent of $z$. After the interchange of the order of summation and integration, the independence with respect to $z$ translates into a remainder that can be bounded independently of $z$ in a large unbounded region of the complex $z$-plane that contains the point $z=0$ and that we specify in Theorems 2.1 and 3.1 below. In the following section we consider the integral representation (1.1) for $\Re b \leq 1$. In Section 3 we consider the integral representation (1.2) for $\Re b \geq 1$. Section 4 contains some comments about the accuracy of the approximations. Throughout the paper we use the principal $\operatorname{argument} \arg z \in(-\pi, \pi]$.

2. A uniform convergent expansion of $\boldsymbol{B}_{\boldsymbol{z}}(\boldsymbol{a}, \boldsymbol{b})$ for $\Re b \leq 1$. In this section we consider the integral representation (1.1). We define the extended sector (see Figure 2.1):

$$
S_{\theta}:=\{\theta \leq|\arg (z)| \leq \pi\} \cup\left\{z \in \mathbb{C} ;\left|z-\frac{1}{2}\right| \leq \frac{1}{2} \text { and }|z-1| \geq \sin \theta\right\}
$$


with arbitrary $0<\theta \leq \pi / 2$. We have the following theorem:

THEOREM 2.1. For $\Re a>0, \Re b \leq 1, z \in S_{\theta}$, with $0<\theta \leq \pi / 2$, and $n=1,2,3, \ldots$,

$$
z^{-a} B_{z}(a, b)=2^{1-a} \sum_{k=0}^{n-1} \frac{(1-a)_{k}}{k !} \beta_{k}(z, b)+R_{n}(z, a, b),
$$

where $\beta_{k}(z, b)$ are the elementary functions

$$
\begin{aligned}
\beta_{k}(z, b):=\frac{1}{z^{k+1}} \sum_{j=0}^{k}\left(\begin{array}{l}
k \\
j
\end{array}\right) 2^{j}(z-2)^{k-j} & \\
& \times\left[\frac{1-(1-z)^{j+b}}{j+b}\left(1-\delta_{j,-b}\right)-\delta_{j,-b} \log (1-z)\right],
\end{aligned}
$$

with $\delta_{k, j}$ the Kronecker delta: $\delta_{k, j}=1$ if $k=j, \delta_{k, j}=0$ if $k \neq j$. In (2.3), the first term inside the brackets must be understood as zero if $j=-b$. For $k=1,2,3, \ldots$ and $b \neq 0$, the coefficients $\beta_{k}(z, b)$ satisfy the recurrence relation

$$
\begin{aligned}
& \beta_{k}(z, b)=\frac{1}{z b}\left[1-(-1)^{k}(1-z)^{b}\right]-\frac{2 k}{z b} \beta_{k-1}(z, b+1), \\
& \beta_{0}(z, b)=\frac{1}{z b}\left[1-(1-z)^{b}\right] .
\end{aligned}
$$

On the other hand, for $k=1,2,3, \ldots$ and $b=0$,

$$
\begin{aligned}
& \beta_{k}(z, 0)=\frac{1-(-1)^{k}}{k z}+\left(1-\frac{2}{z}\right) \beta_{k-1}(z, 0), \\
& \beta_{0}(z, 0)=-\frac{1}{z} \log (1-z) .
\end{aligned}
$$

When $z=0$, the above expressions must be understood in the limit sense. In the extended sector $S_{\theta}$, the remainder is bounded in the form

$$
\left|R_{n}(z, a, b)\right| \leq[\sin (\theta)]^{\Re b-1} \frac{e^{\pi|\Im b|}\left|(1-a)_{n}\right|}{n ! 2^{\Re a-1} \Re a} \max \left\{2^{\Re a-n-1}, 1\right\} .
$$

For $n \geq \Re a-1>0$, the remainder term may also be bounded in the form

$$
\left|R_{n}(z, a, b)\right| \leq[\sin (\theta)]^{\Re b-1} \frac{e^{\pi|\Im b|} 2^{1-\Re a} n\left|(1-a)_{n}\right|}{(n+1) !(\Re a-1)} .
$$

The remainder term behaves as $R_{n}(z, a, b) \sim n^{-\Re a}$ as $n \rightarrow \infty$ uniformly in $|z|$ in the extended sector $S_{\theta}$.

Proof. Consider the truncated Taylor series expansion of the factor $t^{a-1}$ in the integrand of the integral definition (1.1) of $B_{z}(a, b)$ at the middle point $t=1 / 2$ of the integration interval,

$$
t^{a-1}=\frac{1}{2^{a-1}} \sum_{k=0}^{n-1} \frac{(1-a)_{k}}{k !}(1-2 t)^{k}+r_{n}(t, a), \quad t \in(0,1],
$$

where $r_{n}(t, a)$ is the Taylor remainder

$$
r_{n}(t, a):=\frac{1}{2^{a-1}} \sum_{k=n}^{\infty} \frac{(1-a)_{k}}{k !}(1-2 t)^{k}, \quad t \in(0,1] .
$$




\section{ETNA}

Kent State University and

Johann Radon Institute (RICAM)

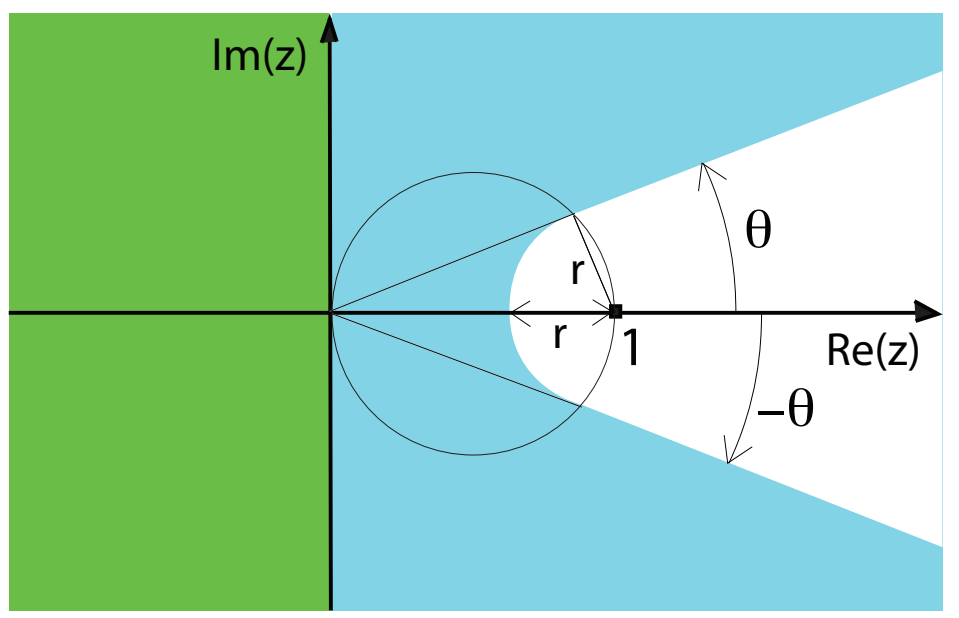

FIG. 2.1. The blue and green regions comprise the extended sector $S_{\theta}$ defined in (2.1), with $r:=\sin \theta$, $0<\theta \leq \pi / 2$. In particular, $S_{\pi / 2}$ is just the half plane $\Re z \leq 0$ and $\lim _{\theta \rightarrow 0} S_{\theta}=\mathbb{C} \backslash[1, \infty)$. In the region $S_{\theta}$, the remainder $R_{n}(z, a, b)$ is bounded independently of $|z|$ by the right-hand side of (2.6).

Instead of the point $t=1 / 2$ for the Taylor expansion of $t^{a-1}$, we could have chosen any other point $t \in[1 / 2,1]$ such that the convergence disk of the Taylor series of $t^{a-1}$ contains the interval $[0,1]$. We have selected the point $t=1 / 2$ because in a previous paper [1], where we analyzed a similar problem for the incomplete gamma function, we proved that the optimal choice was the middle point of the interval.

After suitable manipulations, we can write

$$
r_{n}(t, a)=\frac{(1-a)_{n}}{2^{a-1} n !}(1-2 t)^{n}{ }_{2} F_{1}(n+1-a ; n+1 ; 1-2 t), \quad t \in(0,1] .
$$

Replacing (2.8) in the integral representation of $B_{z}(a, b)$ given in (1.1) and interchanging the order of summation and integration, we obtain (2.2) with

$$
R_{n}(z, a, b):=\int_{0}^{1} r_{n}(t, a)(1-z t)^{b-1} d t
$$

and

$$
\beta_{k}(z, b):=\int_{0}^{1}(1-2 t)^{k}(1-z t)^{b-1} d t=\frac{1}{z} \int_{1-z}^{1}\left(1-\frac{2}{z}+2 \frac{u}{z}\right)^{k} u^{b-1} d u .
$$

Expanding the first factor of the integrand in the second integral in terms of powers of $u$ and integrating term-wise, we obtain (2.3). Integrating by parts in any of the integrals in (2.12), it is straightforward to see that, for $k=1,2,3, \ldots$, the functions $\beta_{k}(z, b)$ satisfy the recurrence relations (2.4) and (2.5).

In order to derive the bound (2.6), we need a bound for the factor $(1-z t)^{b-1}$ uniformly valid for $t \in[0,1]$. It is straightforward to verify that, for $t \in[0,1]$, we have the bound $\left|(1-z t)^{b-1}\right| \leq e^{\pi|\Im b|} M(z, b)$ with

$$
M(z, b):= \begin{cases}1, & \text { if } \Re(z) \leq 0, \\ |1-z|^{\Re b-1}, & \text { if } \Re(1 / z) \geq 1, \\ |\sin (\arg (z))|^{\Re b-1}, & \text { if } 0<\Re(1 / z)<1 .\end{cases}
$$




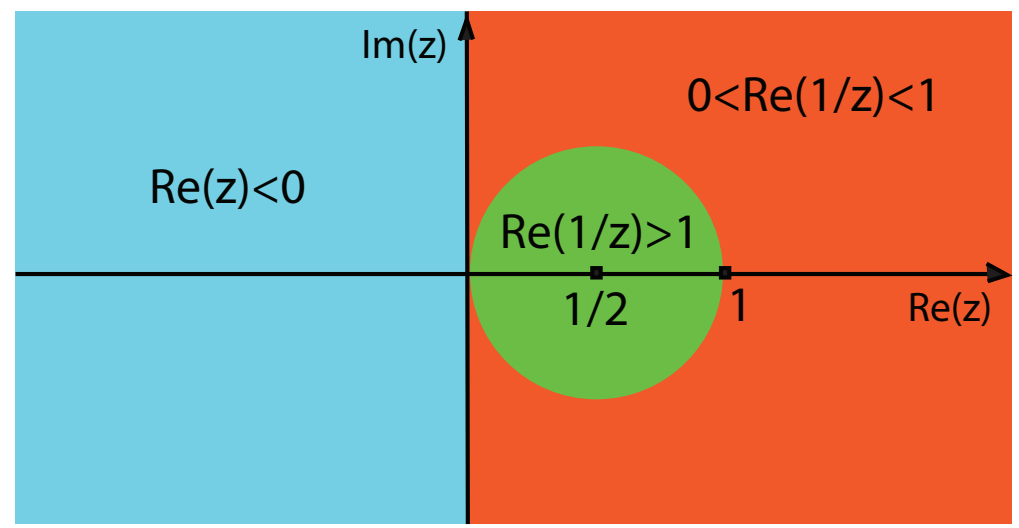

FIG. 2.2. Different regions considered in formula (2.13). The green region $\Re(1 / z)>1$ is the open disk of radius $1 / 2$ with center at $z=1 / 2$. The red region $0<\Re(1 / z)<1$ is the intersection of the half plane $\Re z>0$ with the exterior of this disk.

The regions of the complex $z$-plane considered in this formula are depicted in Figure 2.2. For $z \in S_{\theta}$, with $0<\theta \leq \pi / 2$, we have that $M(z, b) \leq[\sin (\theta)]^{\Re b-1}$. This inequality may be proved by using the following geometrical arguments:

(i) at the points of the circle $|z-1 / 2|=1 / 2$, we have that $|1-z|=|\sin (\arg (z))|$;

(ii) the closest points of the sector $\theta \leq|\arg (z)|<\pi / 2$ to the point $z=1$ are just the two points obtained from the intersection of the rays $\arg z= \pm \theta$ with the circle $|z-1 / 2|=1 / 2$

(iii) the closest points of the region $\left\{z \in \mathbb{C} ;\left|z-\frac{1}{2}\right| \leq \frac{1}{2}\right.$ and $\left.|z-1| \geq \sin \theta\right\}$ to the point $z=1$ are those of the portion of the circle $|z-1|=\sin \theta$ contained inside this region.

Now we use that $r_{n}(t, a)$ is integrable in $(0,1)$, the bound $\left|(1-z t)^{b-1}\right| \leq e^{\pi|\Im b|}[\sin (\theta)]^{\Re b-1}$ for $t \in[0,1]$, and insert (2.10) into (2.11). Thus, we obtain

$$
\begin{aligned}
\left|R_{n}(z, a, b)\right| \leq e^{\pi|\Im b|}[\sin (\theta)]^{\Re b-1} \frac{\left|(1-a)_{n}\right|}{n ! 2^{\Re a-1}} & \\
& \times \int_{0}^{1}|1-2 t|^{n}\left|{ }_{2} F_{1}(n+1-a, 1 ; n+1 ; 1-2 t)\right| d t .
\end{aligned}
$$

From the integral representation of the hypergeometric function [8, eq. (15.6.1)], we find that, for $t \in(0,1)$,

$$
\left|{ }_{2} F_{1}(n+1-a, 1 ; n+1 ; 1-2 t)\right| \leq{ }_{2} F_{1}(n+1-\Re a, 1 ; n+1 ; 1-2 t) .
$$

Then,

$$
\begin{aligned}
& \left|R_{n}(a, z, b)\right| \leq e^{\pi|\Im b|}[\sin (\theta)]^{\Re b-1} \frac{\left|(1-a)_{n}\right|}{n ! 2^{\Re a-1}} \\
& \quad \times \int_{0}^{1}|1-2 t|^{n} F_{1}(n+1-\Re a, 1 ; n+1 ; 1-2 t) d t \\
& =e^{\pi|\Im b|}[\sin (\theta)]^{\Re b-1} \frac{\left|(1-a)_{n}\right|}{n ! 2^{\Re a}}\left[\frac{1}{\Re a}+\frac{1}{n+1}{ }_{2} F_{1}(n+1-\Re a, 1 ; n+2 ;-1)\right] .
\end{aligned}
$$


Using now the contiguous hypergeometric function [8, eq. (15.5.14)] with $a=1$, $b=n+1-\Re a, c=n+1$, and $z=-1$, we find that

$$
\begin{aligned}
\frac{1}{n+1}{ }_{2} F_{1}(n+1-\Re a, 1 ; n+2 ;-1)= & \frac{\Re a+1}{n \Re a}{ }_{2} F_{1}(n+1-\Re a, 1 ; n+1 ;-1) \\
& -\frac{2}{n \Re a}{ }_{2} F_{1}(n+1-\Re a, 2 ; n+1 ;-1),
\end{aligned}
$$

and by applying [8, eq. (15.5.11)] in the second hypergeometric function, we can write

$$
2{ }_{2} F_{1}(n+1-\Re a, 2 ; n+1 ;-1)=n+(\Re a+1-2 n)_{2} F_{1}(n+1-\Re a, 1 ; n+1 ;-1) .
$$

Thus, inserting these formulas into (2.14), we get

$$
\left|R_{n}(z, a, b)\right| \leq e^{\pi|\Im b|}[\sin (\theta)]^{\Re b-1} \frac{\left|(1-a)_{n}\right|}{n ! 2^{\Re a-1} \Re a}{ }_{2} F_{1}(n+1-\Re a, 1 ; n+1 ;-1) .
$$

From the integral representation of the hypergeometric function [8, eq. (15.6.1)], we have that

$$
{ }_{2} F_{1}(n+1-\Re a, 1 ; n+1 ;-1)=n \int_{0}^{1}(1-t)^{n-1}(1+t)^{\Re a-n-1} d t \leq \max \left\{2^{\Re a-n-1}, 1\right\} .
$$

The bound (2.6) follows from (2.15) and this last inequality.

When $n \geq \Re a-1>0$, we consider again the integral representation of the hypergeometric function [8, eq. (15.6.1)],

$$
\left|{ }_{2} F_{1}(n+1-a, 1 ; n+1 ; 1-2 t)\right| \leq n \int_{0}^{1}(1-s)^{n-1}[1-(1-2 t) s]^{\Re a-n-1} d s .
$$

When $t \in(0,1)$, we have $[1-(1-2 t) s]^{\Re a-n-1} \leq(1-s)^{\Re a-n-1}$, and thus,

$$
\left|{ }_{2} F_{1}(n+1-a, 1 ; n+1 ; 1-2 t)\right| \leq \frac{n}{\Re a-1} .
$$

Therefore, from (2.10) we have that

$$
\left|r_{n}(t, a)\right| \leq \frac{\left|(1-a)_{n}\right||1-2 t|^{n}}{2^{\Re a-1}(n-1) !(\Re a-1)} .
$$

Formula (2.7) follows straightforwardly by inserting this bound into (2.11).

Finally, using the Stirling formula and [6, eq. (30)] in (2.6) or (2.7), we obtain that $R_{n}(a, z) \sim n^{-\Re a}$ as $n \rightarrow \infty$. Then, any of the bounds (2.6) or (2.7) show the uniform character of the expansion (2.2) in the extended sector $S_{\theta}$.

Formula (1.6) follows from Theorem 2.1 with $a=5 / 2, b=1 / 2$, and $n=3$. An error bound simpler than those given in (2.6) and (2.7) can be found when $a$ is real. It is stated in the following proposition.

PROPOSITION 2.2. For $a>0, \Re b \leq 1, z \in S_{\theta}$, and $n=1,2,3, \ldots$, the error term $R_{n}(z, a, b)$ in Theorem 2.1 may be bounded as

$$
\left|R_{n}(z, a, b)\right| \leq[\sin (\theta)]^{\Re b-1} \frac{e^{\pi|\Im b|}\left|(1-a)_{n}\right|}{2^{a-1} a n !} .
$$

Proof. Take $p:=\lfloor a\rfloor$, and define $\alpha:=a-p$. Then we have that, for $k \geq p$,

$$
(1-a)_{k}=(-1)^{p}(\alpha)_{p}(1-\alpha)_{k-p} .
$$


TABLE 2.1

The first few terms in the expansion (2.2) of $z^{-a} B_{z}(a, b)$ when $-b \notin \mathbb{N} \cup\{0\}$.

\begin{tabular}{c|c}
$n$ & $(1-a)_{n} \beta_{n}(z, b) / n !$ \\
\hline 0 & $\frac{\left(1-(1-z)^{b}\right)}{b z}$ \\
1 & $\frac{\left((a-2)(1+b)+(a-2-a b)(1-z)^{b}\right)}{b(b+1) z}+\frac{2(a-1)\left(1-(1-z)^{b}\right)}{b(b+1) z^{2}}$ \\
2 & $\frac{\left((-3+a)(-2+a)(1+b)(2+b)+\left(a(10+(-5+b) b)+a^{2}\left(-2+b-b^{2}\right)-2\left(6+b+b^{2}\right)\right)(1-z)^{b}\right)}{2 b(b+1)(b+2) z}$ \\
& $+\frac{2(-1+a)\left(-(-3+a)(2+b)+(-6-a(-2+b)+b)(1-z)^{b}\right)}{b(b+1)(b+2) z^{2}}+\frac{4(-2+a)(-1+a)\left(1-(1-z)^{b}\right)}{b(b+1)(b+2) z^{3}}$
\end{tabular}

Using this equality in (2.9), we find that

$$
\left|r_{n}(t, a)\right| \leq \frac{(\alpha)_{p}}{2^{a-1}} \sum_{k=n}^{\infty} \frac{(1-\alpha)_{k-p}}{k !}|1-2 t|^{k} .
$$

We insert this bound into (2.11), and by using that $\left|(1-z t)^{b-1}\right| \leq e^{\pi|\Im b|}[\sin (\theta)]^{\Re b-1}$ for $t \in[0,1]$ and (2.17), we find (2.16).

Table 2.1 provides the first few terms of the approximation of $z^{-a} B_{z}(a, b)$ given by the expansion (2.2) for $\Re b \leq 1$ and $-b \notin \mathbb{N} \cup\{0\}$. These terms are rational functions of $z$ and functions of $(1-z)^{b}$. When $-b \in \mathbb{N} \cup\{0\}$, the terms of the expansion (2.2) also contain the term $\log (1-z)$.

In Figure 2.3 we plot the relative errors, on a logarithmic scale, of the approximation of $z^{-a} B_{z}(a, b)$ given in Theorem 2.1 for $n=1,2,3,4,5, z=\rho e^{i \theta}$, with $\theta=0, \pi / 4, \pi / 2,-\pi / 3$, $\rho \in[-100,1)$ or $\rho \in[-100,100]$, and different values of the parameters $a$ and $b$. This serves as a numerical experiment investigating the rate of convergence provided by (2.2). We also observe the uniform character of the approximation in the region $S_{\theta}$.

3. A uniform convergent expansion of $\boldsymbol{B}_{z}(a, b)$ for $\Re b \geq 1$. In this section we consider the integral representation (1.2). For any $0<r \leq 1$, consider the punctured complex plane at $z=1$ with the interval $[1, \infty)$ removed:

$$
C_{r}:=\{z \in \mathbb{C} ;|z-1| \geq r,|\arg (1-z)|<\pi\} .
$$

We have the following theorem.

THEOREM 3.1. For $\Re a>0, \Re b \geq 1, z \in C_{r}$, with $0<r \leq 1$, and $n=1,2,3, \ldots$,

$$
z^{-a}(1-z)^{1-b} B_{z}(a, b)=2^{1-a} \sum_{k=0}^{n-1} \frac{(-1)^{k}(1-a)_{k}}{k !} \beta_{k}(z, b)+R_{n}(z, a, b),
$$

where the functions $\beta_{k}(z, b)$ are the elementary functions

$$
\beta_{k}(z, b):=\frac{1}{z^{k+1}} \sum_{j=0}^{k}\left(\begin{array}{l}
k \\
j
\end{array}\right)(-2)^{j}(2-z)^{k-j} \frac{(1-z)^{1-b}-(1-z)^{j+1}}{j+b} .
$$



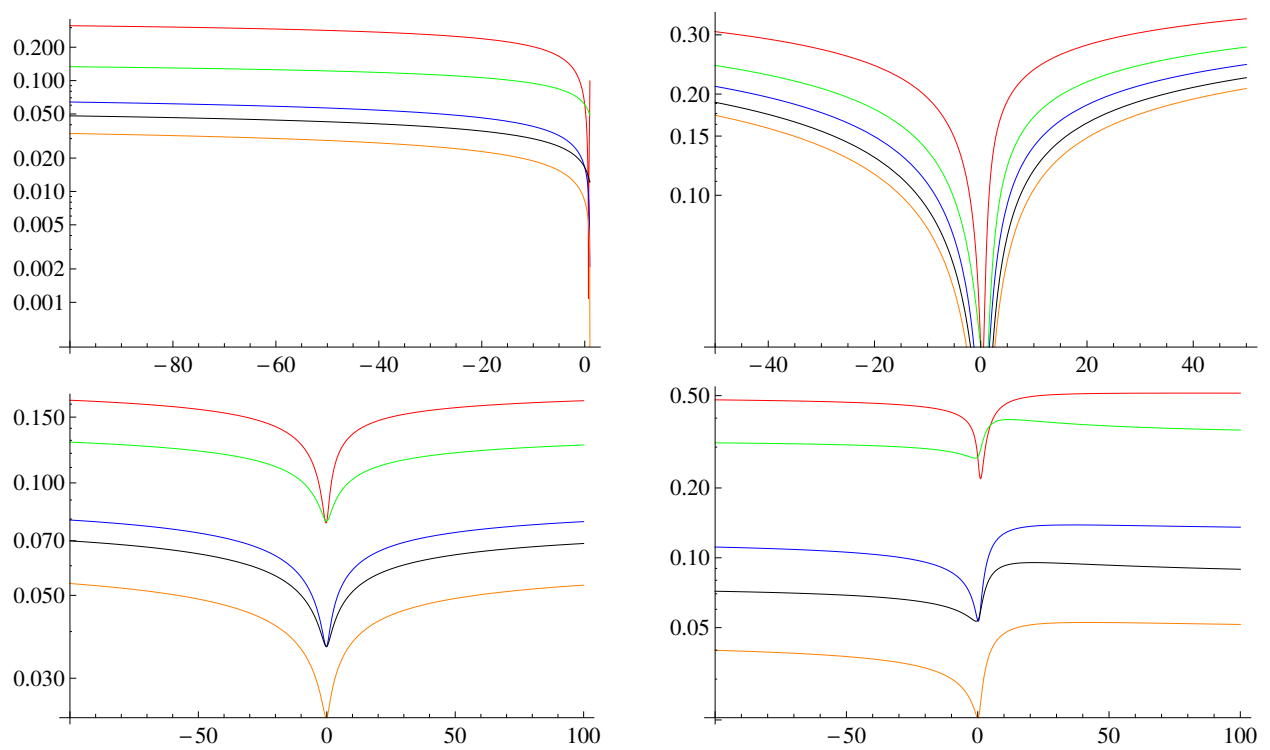

FIG. 2.3. Plots of the relative errors, on a logarithmic scale, of the approximation of $z^{-a} B_{z}(a, b)$ given in Theorem 2.1 for $n=1$ (red), $n=2$ (green), $n=3$ (blue), $n=4$ (black), and $n=5$ (orange) for $z=\rho e^{i \theta}$ with $\theta=0, a=1.5, b=0.5$ (top left), $\theta=\pi / 4, a=0.9, b=-1-0.3 i$ (top right), $\theta=\pi / 2, a=1+0.25 i, b=0.7$ (bottom left), $\theta=-\pi / 3, a=2.1+i, b=0.8+0.2 i$ (bottom right), and $\rho \in[-100,1)$ or $\rho \in[-100,100]$.

For $k=1,2,3, \ldots$, they satisfy the recurrence relation

$$
\begin{aligned}
& \beta_{k}(z, b)=\frac{1-z}{z b}\left[\frac{(-1)^{k}}{(1-z)^{b}}-1\right]+\frac{2 k(1-z)}{z b} \beta_{k-1}(z, b+1), \\
& \beta_{0}(z, b)=\frac{1-z}{z b}\left[\frac{1}{(1-z)^{b}}-1\right] .
\end{aligned}
$$

When $z=0$, the above expressions must be understood in the limit sense. The remainder is bounded in the form

$$
\left|R_{n}(z, a, b)\right| \leq \frac{e^{\pi|\Im b|}\left|(1-a)_{n}\right|}{n ! 2^{\Re a-1} \Re a r^{\Re b-1}} \max \left\{2^{\Re a-n-1}, 1\right\} .
$$

For $n \geq \Re a-1>0$, the remainder term may also be bounded in the form

$$
\left|R_{n}(z, a, b)\right| \leq \frac{e^{\pi|\Im b|} 2^{1-\Re a}\left|(1-a)_{n}\right|}{(n-1) !(n+1)(\Re a-1) r^{\Re b-1}} .
$$

The remainder term behaves as $R_{n}(z, a, b) \sim n^{-\Re a}$ as $n \rightarrow \infty$, uniformly for $z \in C_{r}$.

Proof. The proof is similar to that of Theorem 2.1 but considering the integral representation (1.2) instead of (1.1). That is, we must consider the Taylor expansion of the factor $(1-t)^{a-1}$ at $t=1 / 2$ instead of the expansion of the factor $t^{a-1}$. Moreover, we must replace $z$ by $z /(z-1)$ in the factor $(1-z t)^{b-1}$. We only give here a few significant details.

Replacing the truncated Taylor series expansion of $(1-t)^{a-1}$ at $t=1 / 2$ on the right-hand side of (1.2), we obtain (3.2) with

$$
R_{n}(z, a, b):=\int_{0}^{1} r_{n}(t, a)\left(1+\frac{z}{1-z} t\right)^{b-1} d t
$$


and

$$
\begin{aligned}
\beta_{k}(z, b): & =\int_{0}^{1}(1-2 t)^{k}\left(1+\frac{z}{1-z} t\right)^{b-1} d t \\
& =\frac{1-z}{z} \int_{1}^{(1-z)^{-1}}\left(\frac{2-z}{z}-\frac{2(1-z)}{z} u\right)^{k} u^{b-1} d u .
\end{aligned}
$$

Expanding the first factor of the integrand in the second integral in terms of powers of $u$ and integrating term-wise, we obtain (3.3). Thus, we obtain (3.2) with $R_{n}(z, a, b)$ given in (3.7). Now, in order to derive the bounds (3.5) and (3.6) instead of a bound for the factor $(1-z t)^{b-1}$ valid for every $t \in[0,1]$, we need a bound for the factor $\left(1-z(z-1)^{-1} t\right)^{b-1}$ valid for every $t \in[0,1]$. It is given by $\left|\left(1-z(z-1)^{-1} t\right)^{b-1}\right| \leq e^{\pi|\Im b|} \bar{M}(z, b)$ with

$$
\bar{M}(z, b):=\max \left\{1,|1-z|^{1-\Re b}\right\} .
$$

It is clear that $\bar{M}(z, b) \leq r^{1-\Re b}$, for $z \in C_{r}$, and thus, instead of (2.6) and (2.7), we obtain (3.5) and (3.6).

Numerical experiments show that the recurrence relations (2.4) and (3.4) are not stable for general values of the variable $z$ and the parameter $b$. Therefore, for large values of $k$, it is more convenient to use the explicit expressions (2.3) or (3.3), respectively.

Simpler error bounds than (3.5) and (3.6) can be found when $a$ is real. The proof is similar to the proof of Proposition 2.2, and we omit it.

Proposition 3.2. For $a>0, \Re b \geq 1, z \in C_{r}$, with $C_{r}$ defined in (3.1), for $0<r \leq 1$ and $n=1,2,3, \ldots$, the error term $R_{n}(z, a, b)$ defined by (3.7) in Theorem 3.1 may be bounded in the form

$$
\left|R_{n}(z, a, b)\right| \leq \frac{e^{\pi|\Im b|}\left|(1-a)_{n}\right|}{a r^{\Re b-1} 2^{a-1} n !} .
$$

Table 3.1 shows the first few terms of the approximation of $z^{-a}(1-z)^{1-b} B_{z}(a, b)$ given by the expansion (3.2). These terms are rational functions of $z$ and functions of $(1-z)^{b}$. In Figure 3.1 we give the relative errors, on a logarithmic scale, of the approximation of $z^{-a}(1-z)^{1-b} B_{z}(a, b)$ from Theorem 3.1 for $n=1,2,3,4,5$ and $z=\rho e^{i \theta}$ with $\theta=0, \pi / 4, \pi / 2,-\pi / 3, \rho \in[-100,1)$ or $\rho \in[-100,100]$, and different values of the parameters $a$ and $b$. This serves as a numerical experiment confirming the rate of convergence provided by (3.2). We also observe the uniform character of the approximation in the region $C_{r}$.

4. Some remarks on the numerical experiments. The numerical experiments performed for large values of the parameters $|a|$ and/or $|b|$ show that the accuracy of the approximation (2.2) gets worse as the values of the parameters become larger (one or both). For the approximation (3.2), the relative errors grow for large $|a|$ or large $|a|$ and $|b|$, although the precision is acceptable for large $|b|$, except for values of $z \in[0,2]$, approximately. So, both approaches get worse in general with increasing $|a|$ and/or $|b|$. In order to avoid these numerical difficulties when one of these parameter is large, it is possible to apply the recurrence relations [9, eq. 8.17.16, eq. 8.17.17, eq. 8.17.20 or eq. 8.17.21] to replace them by smaller ones and then use the expansions (2.2) and (3.2). The new approximations obtained in this way remain expressions in terms of elementary functions. Of course, this approach can be applied as long as one is sure about the numerical stability of the above mentioned recursions.

On the other hand, numerical experiments indicate that the error bounds (2.6), (2.7), (3.5), and (3.6) oscillate between values of two and three times the maximum error in the corresponding approximation, depending on the parameters: the bounds are better for small values of the parameters $a$ and $b$. 
TABLE 3.1

The first few terms in the expansion (3.2) of $z^{-a}(1-z)^{1-b} B_{z}(a, b)$.

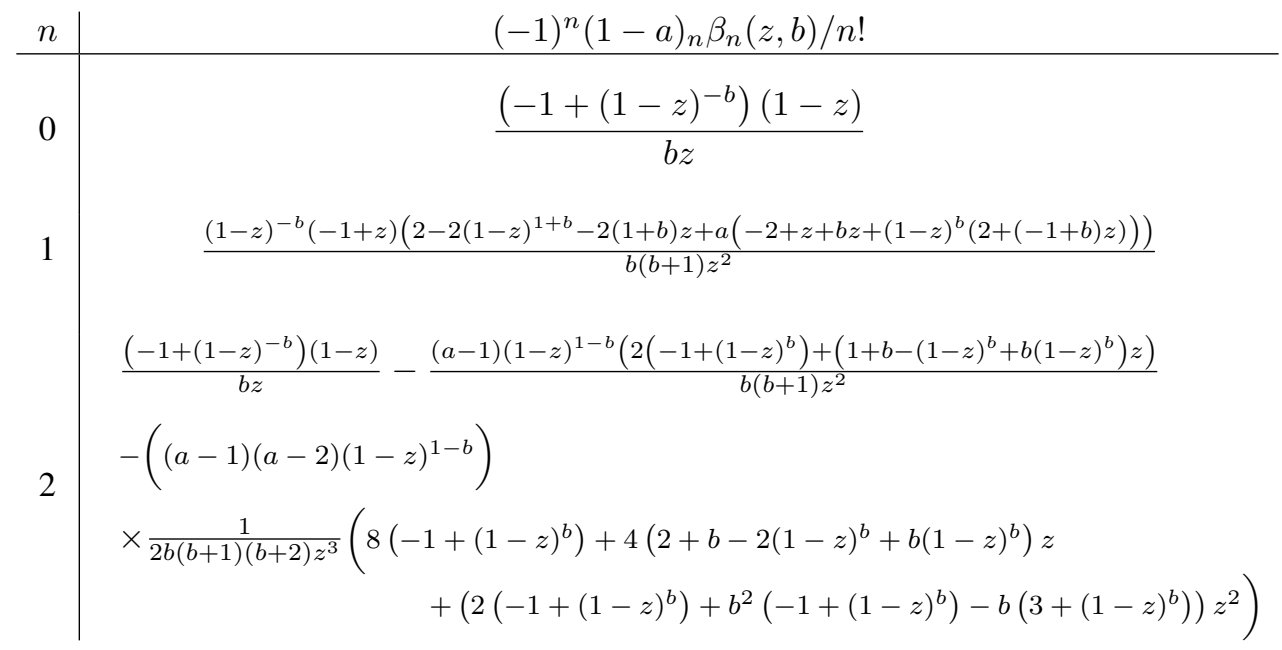
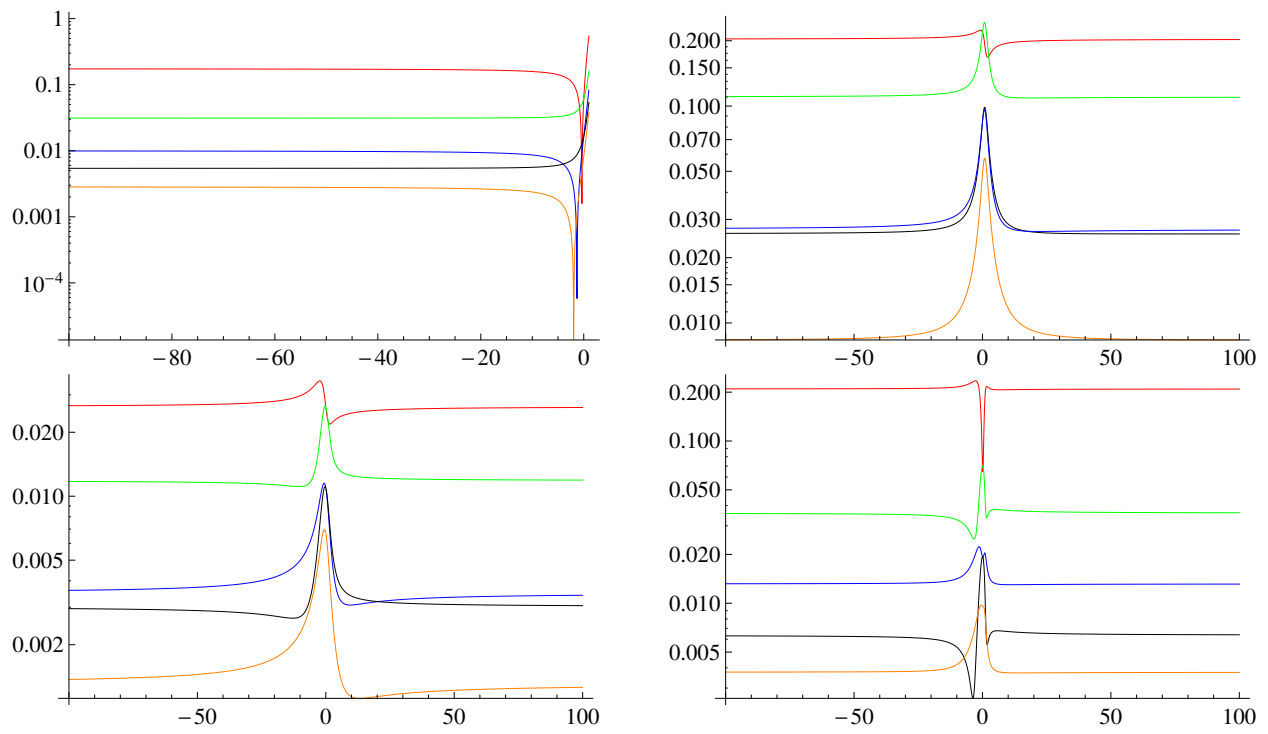

FIG. 3.1. Plots of the relative errors, on a logarithmic scale, of the approximation of $z^{-a}(1-z)^{1-b} B_{z}(a, b)$ given in Theorem 3.1 for $n=1$ (red), $n=2$ (green), $n=3$ (blue), $n=4$ (black), and $n=5$ (orange) for $z=\rho e^{i \theta}$ with $\theta=0, a=1.5, b=3$ (top left), $\theta=\pi / 4, a=1.3+0.75 i, b=2$ (top right), $\theta=\pi / 2, a=1.1$, $b=2.25+0.25 i$ (bottom left), $\theta=-\pi / 3, a=1.5-0.2 i, b=3.0-i$ (bottom right), and $\rho \in[-100,1)$ or $\rho \in[-100,100]$.

Acknowledgments. This research was supported by Ministerio de Economía, Industria y Competitividad, Gobierno de España, project MTM2017-83490-P, Gobierno de Aragón and European Social Fund (group E24_17R).

\section{REFERENCES}

[1] B. BujAnda, J. L. LÓPEZ, AND P. J. PAGOLA, Convergent expansions of the incomplete gamma functions in 
ETNA

Kent State University and

Johann Radon Institute (RICAM)

UNIFORM REPRESENTATIONS OF THE INCOMPLETE BETA FUNCTION

terms of elementary functions, Anal. Appl. (Singap.), 16 (2018), pp. 435-448.

[2] A. J. S. HAMILTON, Formulae for growth factors in expanding universes containing matter and a cosmological constant, Monthly Notices Roy. Astronom. Soc., 322 (2001), pp. 419-425.

[3] N. L. Johnson, S. Kotz, And N. Balakrishnan, Continuous Univariate Distributions. Vol. 2., 2nd ed., Wiley, New York, 1995.

[4] D. A. KofKe, Comment on "the incomplete beta function law for parallel tempering sampling of classical canonical systems" [J. Chem. Phys. 120 (2004) 4119], J. Chem. Phys., 121 (2004), p. 1167.

[5] J. L. LóPEZ, Convergent expansions of the Bessel functions in terms of elementary functions, Adv. Comput. Math., 44 (2018), pp. 277-294.

[6] J. L. López, P. J. PAgOlA, AND E. PÉRez Sinusía, A simplification of Laplace's method: applications to the gamma function and the Gauss hypergeometric function, J. Approx. Theory, 161 (2009), pp. $280-291$.

[7] G. NEMES AND A. B. Olde DAAlHuis, Uniform asymptotic expansion for the incomplete beta function, SIGMA Symmetry Integrability Geom. Methods Appl., 12 (2016), Art. 101 (5 pages).

[8] A. B. Olde DAalhuis, Hypergeometric function, in NIST Handbook of Mathematical Functions, F. W. J. Olver, D. W. Lozier, R. F. Boisvert, and C. W. Clark, eds., Cambridge University Press, Cambridge, 2010, pp. 383-402.

[9] R. B. PARIS, Incomplete gamma functions, in NIST Handbook of Mathematical Functions, F. W. J. Olver, D. W. Lozier, R. F. Boisvert, and C. W. Clark, eds., Cambridge University Press, Cambridge, 2010, ch. 8, pp. 173-192.

[10] K. Pearson, Tables of Incomplete Beta Functions, 2nd ed., Cambridge University Press, Cambridge, 1968.

[11] N. M. TEMmE, Uniform asymptotic expansions of the incomplete gamma functions and the incomplete beta function, Math. Comp., 29 (1975), pp. 1109-1114. 